\title{
3D SHAPE MEASURING INSTRUMENTS USING HIGH STIFFNESS VIBRATION TOUCH SENSOR
}

\author{
Takanori Miyoshi* Yoji Masui* \\ Kazuhiko Enokishima* Kazuhiko Terashima* \\ * Toyohashi University of Technology \\ 1-1 Hibarigaoka, Tempaku-cho \\ Toyohashi City, 441-8580 \\ Japan
}

\begin{abstract}
In this paper, a novel method to determine the shape of a $3 \mathrm{D}$ object is proposed. Instead of a flexible conventional touch probe, a high-stiffness vibration touch sensor was developed. The proposed sensor consisted of a piezo-electric device and thin probe, and generated feedback voltage according to the relative distance from the object. The structure of this sensor is simple, so that it can withstand high rates of acceleration during the measurement process. A nonlinear synchronous multi-axis control algorithm is also proposed. The effectiveness of our proposed sensor and control algorithm is demonstrated through experiments and simulations. Copyright (c)2005 IFAC
\end{abstract}

Keywords: Sensor systems, Computer-aided testing, Continuous path control, Nonlinear control system, Shape discrimination, Automatic control, Industry automation

\section{INTRODUCTION}

Computer Aided Testing (CAT) is a very important technology for the manufacturing industry, because it acts as the final stage of the manufacturing process beginning with design, followed by production, and concluding with quality control by testing. However, conventional coordinate measuring instruments are prohibitively expensive; moreover, such instruments take a long time to examine a single object. Therefore, complete testing has been impossible for cheap, mass-produced goods such as those manufactured by casting, molding and press. Thus, the ultimate purpose of our research is to develop fast and inexpensive instruments for $3 \mathrm{D}$ shape and/or coordinate measurements.

Generally, 3D shape-measuring instruments can be classified into two types: a touch type, such as the touch trigger probe, and a non-touch type, such as that consisting of a laser sensor and a CCD device. The former is extremely precise, but its measurements are time consuming because the probe's structure is complicated; furthermore, its flexibility prohibits movement of the probe at high rates of acceleration. Meanwhile, the latter can measure an object very quickly, but the color and/or the shape of the object is limited due to diffused reflection and/or the angle of reflection.

In order to solve these problems, we herein propose a novel vibration touch sensor consisting of a piezo-electric device, and plan to develop a $3 \mathrm{D}$ shape-measuring instruments to scan the surface of 3D objects(K. Enokishima and Terashima, 2004). Our proposed sensor has a simple structure and a high degree of stiffness so that it resists motion at high rates of acceleration, Thereby making very fast measurement possible. Furthermore, it is not affected by the color and/or the gloss of an object since it is a touch-type sensor.

To date, many researchers have reported on piezoelectric devices in the field of control and measurement engineering. However, most of these 
studies have focused either on actuators such as those employed for precise positioning and active damping(Sacconi A. and W., 1999; Shuo Hung Chang and Chien, 1999; Kakatcioglu S. and H., 1997) or on sensors such as those that measure pressure and force(T. and J., 1997; Shimizu T. and K., 2002). However, our proposed sensor has characteristics of both an actuator that generates vibration and a sensor that can detect touch; i.e., it is a bilateral vibration touch sensor.

In their research regarding the bilaterality of a piezo-electric device, Baqlio et al. have developed a system for recognizing the nature of an object's surface(Baglio S. and N., 2002). They use two piezo-electric devices: one stimulates the surface of the material, and the other detects the response from the object to this stimulation. Their system detects the nature of a material, e.g., whether glass, plastic, or wood. However, we use only a single piezo-electric device whose purpose is to detect the existence of an object.

In this paper, the control method for our measuring instruments is also discussed. The vibrating sensor is moved toward the object while it vibrates, and it identifies the position of the object by detecting the cessation of vibration caused by the sensor touching the object' surface. Based on this principle, we have developed a commercial product, the PIX-30(Roland. D.G. Co., 2002). However, it took too much time to measure an object because the sensor checked each insignificant grid partitioned within the wide target space.

In order to solve this problem, a new control law is proposed that is expected to produce faster measurements. With it, the sensor moves so as to maintain a steady vibration, scanning the surface of the object continuously. Furthermore, a synchronous multi-axis control algorithm is proposed in order to scan the surface of an object effectively and precisely in cooperation with each other. The effectiveness of our proposed method is demonstrated through experiments and simulations.

\section{STRUCTURE AND SPECIFICATIONS OF THE SENSOR}

\subsection{Structure of the sensor}

The structure of the proposed sensor is shown in Fig.1. A thin probe is attached only to the piezoelectric device. Therefore, the sensor is simple, rigid, and extremely robust. The piezo-electric element consists of two parts. One has a drive electrode and works as the vibrator by applying an alternating current, while the other has a feedback electrode and detects the magnitude of the vibration as an alternating voltage. This sensor plays the roles of both vibrator and sensor.
We defined the $\mathrm{X}$-, $\mathrm{Y}$-, and Z-axis coordinates as shown in Fig.1. The piezo-electric device is a 7BB35-3CA0 made by Murata Corp.
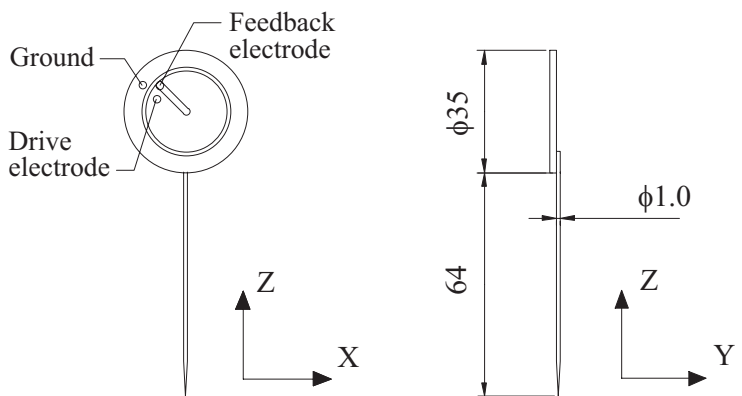

Fig. 1. Structure of the vibration touch sensor

\subsection{Frequency characteristic of the sensor and standing wave on the probe}

The sensor has a natural frequency. Therefore, we had to apply a resonant drive current in order to oscillate the piezo-electric device most efficiently and in order to acquire a large feedback voltage. The frequency characteristics of the feedback voltage when a $0.147[\mathrm{Vp}-\mathrm{p}]$ sinusoidal voltage was applied to the drive electrode are shown in Fig.2.

Without the probe, this piezo-electric device has a $2.58[\mathrm{kHz}]$ natural frequency without a probe(Murata Manufacturing Co., 2003). Note that the resonant frequency moved due to the attachment of the probe. A sinusoidal wave of $2.66[\mathrm{kHz}]$ was selected as the applied voltage. The small peak at $170[\mathrm{~Hz}]$ in this figure demonstrates the probe's first order vibration, in which the resonant frequency is consistent with the result of vibration analysis by ANSYS as shown in Fig.3(a).

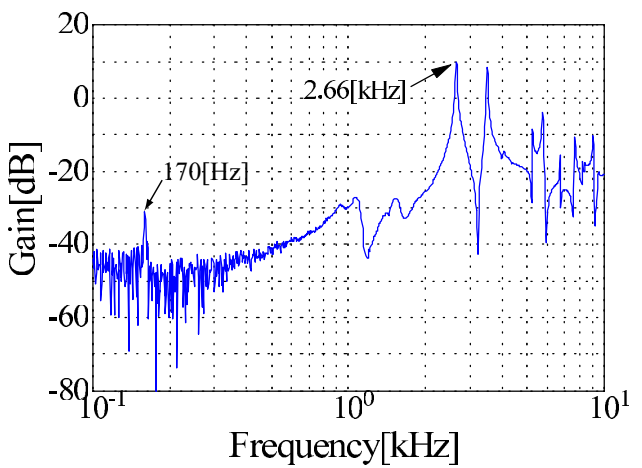

Fig. 2. Frequency characteristics of the feedback voltage

Whereas, the vibration of the multiple-order mode of $2.66[\mathrm{kHz}]$ in Fig.2 is shown in Fig.3(b).

A standing wave with three antinodes and two nodes was observed on the probe according to the position. The phase at the top of the figures and that at the tip of the probe ware consistent with that of the source signal. However, the phase inverse was observed in the third figure. In addition, 


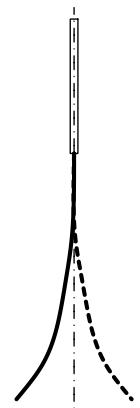

(a) First-order mode (b) Multiple-order mode

Fig. 3. Illustration of multiple-order mode of vibration (Side view)

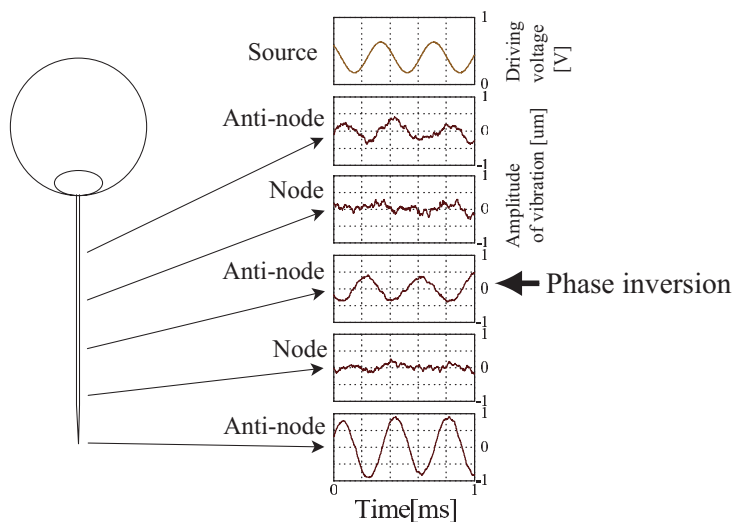

Fig. 4. Standing wave on the probe according to position

two nodes ware observed between the respective anti-node. The process of the transition of the feedback voltage is considered as follows: When the probe touches the object, the tip of the probe stops vibrating. Then, the feedback electrode also becomes a node at the same time that the tip becomes a node of the standing wave. Thus, the feedback voltage decreases.

\subsection{Steady-state characteristic of the sensor}

The bilateral sensor outputs the induced voltage according to the vibration of the probe. The magnitude of the vibration changes according to the relative distance from the object. When the probe is pressed to the object strongly, the vibration stops and the feedback voltage becomes minimal as shown in Fig.5(a). Meanwhile, when the probe vibrates apart from the object, the feedback voltage reaches its maximum as shown in Fig.5(b). When the probe has light contact with the object, the feedback voltage begins to decrease slightly as shown in Fig.5(c). Note that the Fig. 5 is emphasized. Fig. 6 shows the steady state relationship between the relative distance from the sensor to the object with respect to each axis and the feedback voltage when $0.147[\mathrm{Vp}-\mathrm{p}]$ sinusoidal voltage is applied to the drive electrode. The origin on the horizontal axis is the first contact position with the object.

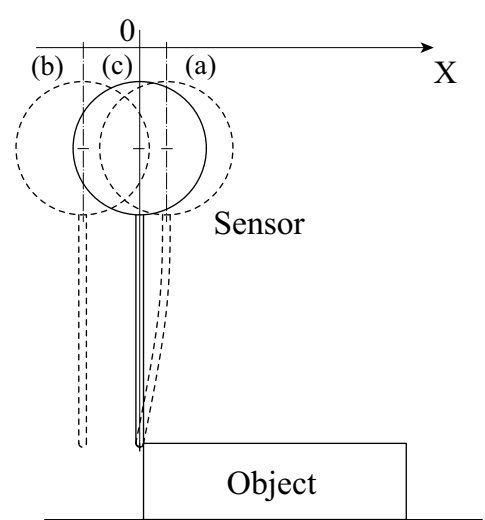

Fig. 5. Illustration of relative distance between the sensor and object
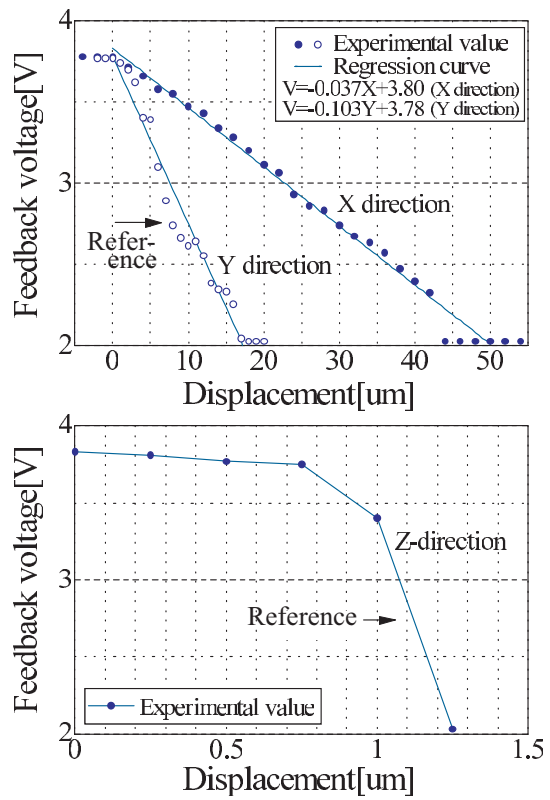

Fig. 6. Relationship between relative distance and feedback voltage

As shown in Fig.6, the transition is approximately linear until saturation due to complete contact. Using this relationship, we can identify not only touch or non-touch but the precise distance from the object. This sensor is especially sensitive with respect to the distance on the Z-axis, having a 0.5 $[\mu \mathrm{m}]$ degree of precision. Thus, we obtained the following equations with respect to the feedback voltage.

$$
\begin{aligned}
& V_{x}= \begin{cases}3.80 & \left(D_{x}<0\right) \\
-0.037 D_{x}+3.80 & \left(0 \leq D_{x} \leq 48.6\right) \\
2.00 & \left(48.6<D_{x}\right)\end{cases} \\
& V_{y}= \begin{cases}3.80 & \left(D_{y}<0\right) \\
-0.103 D_{y}+3.80 & \left(0 \leq D_{y} \leq 36.9\right) \\
2.00 & \left(36.9<D_{x}\right)\end{cases} \\
& V_{z}= \begin{cases}3.80 & \left(D_{z}<0\right) \\
-6.00 D_{z}+3.80 & \left(0 \leq D_{z} \leq 1.2\right) \\
2.00 & \left(1.2<D_{z}\right)\end{cases}
\end{aligned}
$$

where, $V_{x}, V_{y}$, and $V_{z}$ are feedback voltage[V] for the these axis, and $D_{x}, D_{y}$, and $D_{z}$ are the relative 
distance $[\mu \mathrm{m}]$ from the object to the center of the sensor respectively.

\subsection{Robustness for high rates of acceleration}

In order to ensure that the proposed sensor would not encounter trouble during high rates of acceleration motion, we conducted an experiment at approximately $1 \mathrm{G}\left(9.8 \mathrm{~m} / \mathrm{s}^{2}\right)$ acceleration.

The experimental condition were as bellows: Beforehand, we established the origin where the feedback voltage is the reference voltage in Fig.6. We measured the feedback voltage when the sensor moved from the position of $0.1 \mathrm{~m}$ distance from the origin at the maximum speed of $1 \mathrm{~m} / \mathrm{s}$ and $1 \mathrm{G}$ approximately and stopped at the origin. The experimental result is shown in Fig.7. The low level of the positioning signal in the third figure indicates that the sensor exists in the area within $2[\mathrm{um}]$ from the origin. This experimental result shows that the proposed sensor worked correctly in spite of high rates of acceleration and detected the position of the object precisely. Thus, our proposed sensor appears to make high-throughput 3D shape-measuring instruments possible.

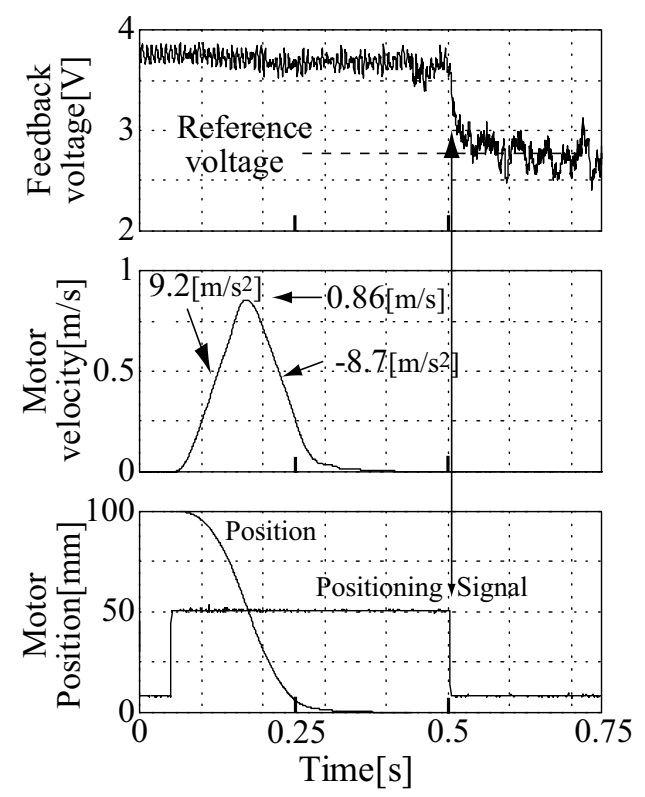

Fig. 7. Feedback voltage during high rates of acceleration

\section{NONLINEAR SYNCHRONOUS CONTROL FOR MEASURING INSTRUMENTS}

\subsection{Experimental apparatus of the measuring instruments}

An X-Y-Z table made by the Roland D.G. Corp. and shown in Fig. 8 was used as the platform of the measuring instruments. This platform moves our proposed sensor attached at the end-effecter toward the X-, Y-, and Z-axes independently. The position of the sensor is controlled at each axis by a stepping motor, and is measured by a laser displacement meter.

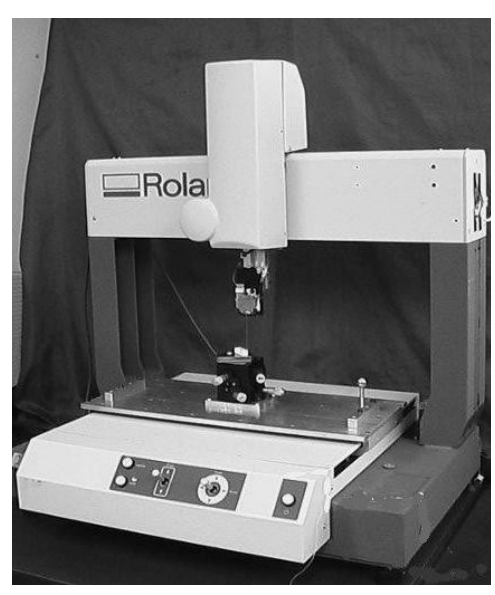

Fig. 8. Experimental apparatus of measuring instruments

\subsection{Independent control with single axis}

As preparation for scanning a 3D shape, each axis was independently controlled as shown in Fig.9.

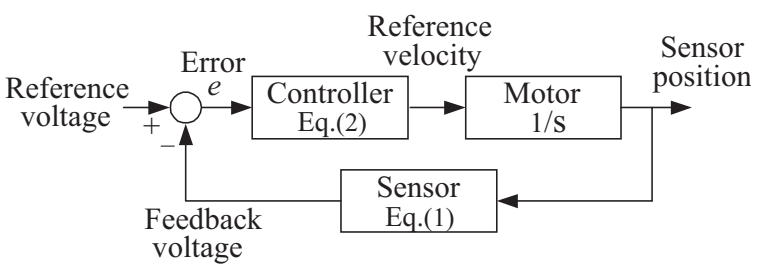

Fig. 9. Block diagram of independent control

The reference voltage is those shown in Fig.6. The position of the sensor was controlled by the motion of the X-Y-Z table, regulating the feedback voltage into the reference voltage so that the sensor was stabilized on the surface of the object. We assumed that the motor moved immediately in accordance with the reference velocity, so that the motor was denoted as a mere integration $1 / s$. The block of the "Sensor" in Fig.9 indicates the function in Eq.(1). The controller was a PI compensator written in Eq.(2).

$$
u=K_{p} e+K_{i} \int e d t
$$

where, $K_{p}$ is the proportional gain, and $K_{i}$ is the integral gain. $e$ is the error between the reference voltage and the feedback voltage, and the control input $u$ is the reference velocity. The simulation and the experimental result show the transient response when the sensor moves close to the object and touches the object.

Figure 10 shows the simulation and experimental results in the $\mathrm{Y}$ axis, and Fig.11 shows them in 


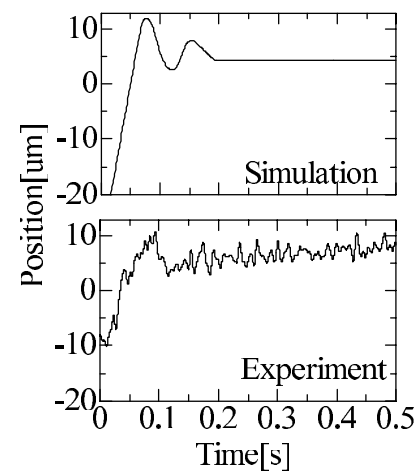

(a) Relative distance

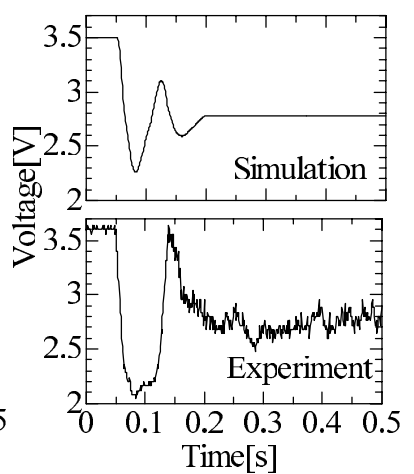

(b) Feedback voltage
Fig. 10. Comparison with simulation and experimental results in $\mathrm{Y}$ axis

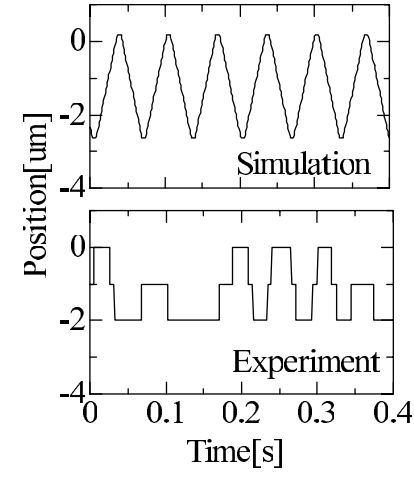

(a) Relative distance

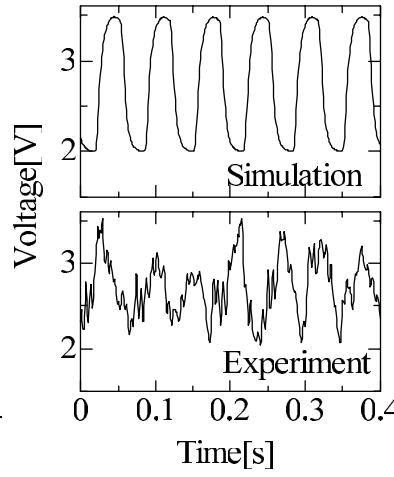

(b) Feedback voltage
Fig. 11. Comparison with simulation and experimental results in $\mathrm{Z}$ axis

the $\mathrm{Z}$ axis. (a) and (b) in those figures illustrate the transition of the relative distance and the feedback voltage of the sensor, respectively. As shown in Fig.10, the experimental results were very similar to the simulation results in regard to overshoot and convergence with the steadystate. In Fig.11, insignificant limit-cycles of 2.0 $[\mu \mathrm{m}]$ were observed in both the simulation and the experimental results. However, those are very similar in regard to period and amplitude. Based on the above results, we concluded that our model described in Eq.(1) and Fig.9 were accurate. In addition, we ensured that our proposed sensor could precisely detected the surface of the object, settling to the relative distance of the reference voltage.

\subsection{Nonlinear synchronous control on two axes}

A block diagram of the synchronous control of the Y- and Z-axes is shown in Fig.12. The control strategy was as follows: The Z-axis was controlled as a master axis by a PI compensator because the $\mathrm{Z}$ axis is so sensitive. On the other hand, the $\mathrm{Y}$ axis was controlled as a slave axis by the cosine of the common integration and another proportional gain. The control law was written in the following equation:

$$
\left\{\begin{array}{l}
u_{z}=K_{p z} e+K_{i z} \int e d t \\
u_{y}=V_{\text {max }} \cos \left(K_{p y} e+\int e d t\right)
\end{array}\right.
$$

where $u_{z}$ and $u_{y}$ are the reference velocities, $V_{\max }$ is the maximum velocity of the $\mathrm{Y}$-axis, $e$ is the error between the reference voltage and the feedback voltage, $K_{p z}$ and $K_{p y}$ are the proportional gain of each axis, and $K_{i z}$ is the integral gain of the Z-axis.

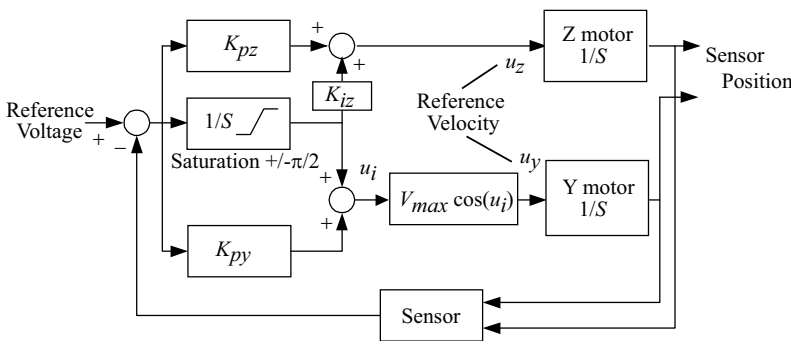

Fig. 12. Block diagram of synchronous control

If the feedback voltage is equal to the reference, then the output of the proportional gain is zero and the integral value is maintained as a steady value. Therefore, the $\mathrm{Y}$ - and $\mathrm{Z}$-axes continue to move at those velocities. Thus, if the object has a linear inclination, then the feedback voltage is consistent with the reference and integral values is fixed in the steady-state, and the velocities of both the Y- and Z-axes maintain a straight-line motion on the object's surface.

On the other hand, in the case that the object has a curved surface, the velocity on the Y-axis may become slow according to the increase of the integration. Note that the integral value becomes saturated up to $\pm \pi / 2$. This means that motion of the $\mathrm{Y}$-axis stops and the only Z-axis moves up or down.

The most difficult condition for surface scanning is a perpendicular shape (It goes without saying that an overhang is beyond the scope of this measurement device). The simulation result for a rectangle $1[\mathrm{~mm}] \times 1[\mathrm{~mm}]$ is shown in Fig.13. The term of the inside of the cosine function is stabilized at $\pm \pi / 2$ at the perpendicular wall. Whereas, the same term is stabilized at 0 at the top of the rectangle. Then, both the error and its integration become zero, and $u_{z}$ and $u_{y}$ are equal to zero and $V_{\max }$, respectively. This means that the sensor moves toward the horizontal direction at maximum speed on the flat top of the rectangle.

In Fig.13, the solid line shows the trajectory of the sensor, and the dotted line shows the contour of the object. The solid line is consistent with the dotted line except for inside the circle. The difference of the trajectory in the circle is caused by the overshoot due to the delay of the control. 


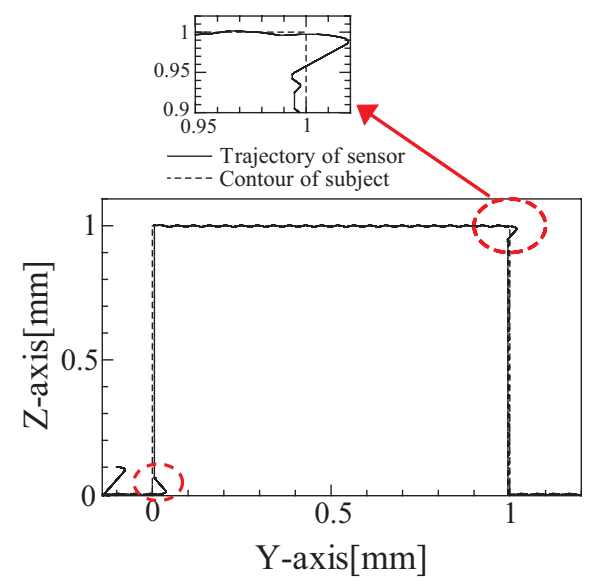

Fig. 13. Simulation result of synchronous control for perpendicular shape

However, this isn't a shortcoming peculiar to this measuring algorithm. Because we can simply reject this part from the measurement results, because the feedback voltage indicates that the sensor is apart from the object. And later, we can measure there again by the same method from the traditional touch-probe. In this simulation, the precision was $8.0[\mu \mathrm{m}]$ on the $\mathrm{Y}$-axis and $5.0[\mu \mathrm{m}]$ on the Z-axis.

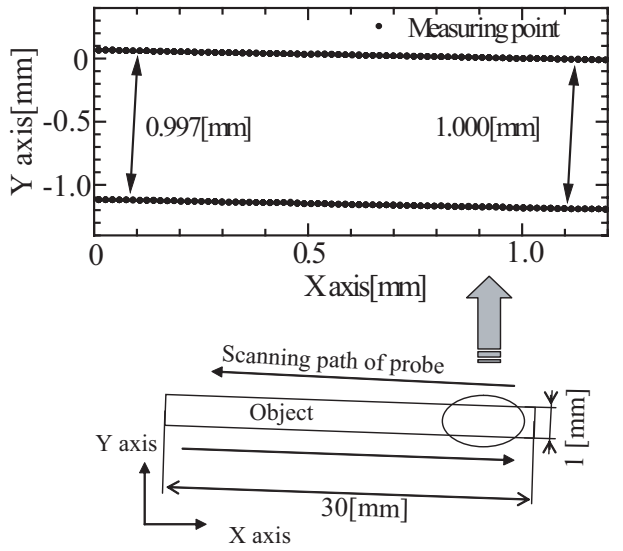

Fig. 14. Experimental result of synchronous control for $\mathrm{X}$-and $\mathrm{Y}$-axes

The experimental results of the synchronous control for the $\mathrm{X}$-and $\mathrm{Y}$-axes are shown in Fig.14. The object was a cera-block of $1[\mathrm{~mm}] \times 30 \mathrm{~mm}$ made by Mitsutoyo Corp. It was placed slantwise in order to demonstrate the effectiveness of the synchronous control. The proposed system determined the width of the object to $3.0[\mu \mathrm{m}]$ degree of precision by considering the width of the probe and the relative distance estimated by the feedback voltage.

\section{CONCLUDING REMARKS}

In this paper, a novel vibration touch sensor and its measurement algorithm were proposed. The proposed sensor consisted of a piezo-electric device and a thin probe, and had high stiffness and robustness against high rates of acceleration, due to the simplicity of the structure. It was determined that the relationship between the feedback voltage and the relative distance from an object is approximately linear. By controlling the sensor position on a single axis, it was demonstrated that the model was accurate and that the proposed system could be stabilized on the surface of the object. Finally, a nonlinear synchronous control was proposed in order to scan the $3 \mathrm{D}$ shape of objects, and its effectiveness was demonstrated through experiments and simulations. The proposed system obtained the contour of a rectangle to $3.0[\mu \mathrm{m}]$ degree of precision in the $\mathrm{X}-\mathrm{Y}$ plane.

\section{REFERENCES}

Baglio S., Muscato G. and Savalli N. (2002). Tactile measuring systems for the recognition of unknown surfaces. IEEE Trans. on Instrumentation and Measurement 51(3), 522-531.

K. Enokishima, Y. Masui, T. Miyoshi and K. Terashima (2004). A method of obtaining shape by bilateral vibration touch sensor. Proc. of SICE Annual Conference pp. 17561759.

Kakatcioglu S., Giray M. and Asmer H. (1997). Vibration control of flexible manipulators using smart structures. Proc. of Int. Symp. on Intelligent Control pp. 415-420.

Murata Manufacturing Co., Ltd. (2003). 7bb35$3 \mathrm{ca} 0$ data book.

Roland. D.G. Co., Ltd. (2002). Pix-30 data book. Sacconi A., Picotto G.B. and Pasin W. (1999). The imgc calibration setup for microdisplacement actuators. IEEE Trans. on Instrumentation and Measurement 48(2), 483-487.

Shimizu T., Shikida M., Sato K. and Itoigawa K. (2002). A new type of tactile sensor detecting contact force and hardness of an object. Proc. of Int. conf. Micro Electro Mechanical Systems pp. 344-347.

Shuo Hung Chang, Chung Kai Tseng and Hon Chan Chien (1999). An ultra-precision $\mathrm{xy} \theta \mathrm{z}$ piezo-micropositioner. ii. experiment and performance. IEEE Trans. on Ultrasonics, Ferroelectrics and Frequency Control.

T., Maudie and Wertz J. (1997). Pressure sensor performance and reliability. Industry Applications Magazine 3(3), 37-43. 